\title{
Structure and Self-Energy of the Electron
}

\author{
S. M. BLINDER \\ Department of Chemistry, University of Michigan, Ann Arbor, Michigan 48109-1055
}

Received 10 April 2001; accepted 2 July 2001

DOI 10.1002/qua.1806

\begin{abstract}
The region very close to an electron $\left(r \lesssim r_{0}=e^{2} / m c^{2} \approx 2.8 \times 10^{-13} \mathrm{~cm}\right)$ is, according to quantum electrodynamics, a seething maelstrom of virtual electron-positron pairs flashing in and out of existence. To take account of this well-established physical reality, a phenomenological representation for vacuum polarization is introduced into the framework of classical electrodynamics. Such a model enables a consistent picture of classical point charges with finite electromagnetic self-energy. (c) 2002 Wiley

Periodicals, Inc. Int J Quantum Chem 90: 144-147, 2002
\end{abstract}

Key words: classical electrodynamics; electron self-energy; point charge; vacuum polarization

$\mathbf{P}$ er-Olov Löwdin was an enthusiastic advocate of the application of the techniques and modes of thought of quantum chemistry to a wide range of scientific problems, including material science, molecular biology, and even particle physics [1]. Inspired by such a fearless spirit of adventure, we propose to go possibly "where no quantum chemist has ever gone" to explore a different vista of the theory of matter-the interior of the electron. This work is dedicated in fond memory to Per-Olov Löwdin for his intellectual inspiration, guidance, and friendship.

Since the discovery of the electron by J. J. Thomson over a century ago [2], a persistant puzzle has been how to account for its electromagnetic selfenergy. This has been the subject of extensive theoretical contemplation by some of the leading figures of twenty century physics [3]. The earliest models (Thomson, Poincaré, Lorentz, Abraham, Schott) [4] pictured the electron as a finite charged sphere,

Correspondence to: S. M. Blinder; e-mail: sblinder@umich.edu. on the scale of the classical electron radius $r_{0}=$ $e^{2} / m c^{2} \approx 2.818 \times 10^{-13} \mathrm{~cm}$. The electromagnetic selfenergy of such a finite structure would be of the order of $W \approx e^{2} / r_{0} \approx m c^{2}$ and thus implies an electron rest mass predominantly electromagnetic in origin. Yet all experimental evidence implies an electron radius much smaller than $r_{0}$, consistent, in fact, with a particle of point mass and point charge [5]. Recent high-energy electron-positron scattering experiments imply an upper limit of $2 \times 10^{-16} \mathrm{~cm}$ on the electron size.

If the electron is indeed a structureless point charge, as assumed in quantum theories, in particular quantum electrodynamics, then how does one avoid the divergent electromagnetic self-energy? An analogous problem is dealt with very elegantly in quantum chemistry. In Hartree-Fock theory, the electrostatic interaction between two spinorbitals $\phi_{\alpha}$ and $\phi_{\beta}$ is given by the difference between the Coulomb and exchange integrals:

$$
J_{\alpha \beta}-K_{\alpha \beta}=\left\langle\alpha, \beta\left|r_{12}^{-1}\right| \alpha, \beta\right\rangle-\left\langle\alpha, \beta\left|r_{12}^{-1}\right| \beta, \alpha\right\rangle .
$$


This reduces to zero when $\alpha=\beta$, thus canceling out all orbital self-interactions. We should like to extend this idea to treat the self-energy of a single electron.

A number of ingenious schemes to avoid a divergent electromagnetic self-energy for a point electron have been proposed over the years by Dirac [6], Wheeler and Feynman [7], Rohrlich [8], Teitelboim [9], and many others. The more recent approaches invoke such arcana as advanced solutions of Maxwell's equations (superposed on the conventional retarded solutions) and/or renormalization of mass and charge infinities. This enables the divergent part of the self-interaction to be avoided while leaving intact the radiation reaction, an effect long known and thoroughly tested.

From a more general perspective, the singularities in fields and energies associated with point charges in classical electrodynamics has been a pervasive flaw in what has been an otherwise beautifully complete and consistent theory. An immense number of attempts to address this problem have been based, roughly speaking, on one of the following lines of argument: (1) Actual point charges do not exist-real particles have a finite size-hence the problem is artificial. (2) By a clever limiting procedure in the formalism, the radius of a charge can be reduced to zero without introducing infinities. (3) Point charges are quantum objects and classical electrodynamics has no business dealing with them. The last point of view, espoused by Frenkel [10] and others, asserts that any classical model is futile because the electron is a quantum mechanical object with no substructure. It is nonetheless of at least academic interest to have a consistent classical relativistic model that connects to macroscopic electrodynamics, while remaining cognizant of its limitations. The purpose of the present study is a modified theory able to handle the singularities produced by point charges while reducing to standard electrodynamics for $r \gg r_{0}$.

We propose to provide possible finishing touches to Maxwell's electromagnetism without making any ad hoc modifications of the fundamental equations of the theory. The key to our approach is the physical reality of vacuum polarization in the submicroscopic vicinity of charged elementary particles.

We will proceed on the premise that the electron rest mass $\left(0.511 \mathrm{MeV} / \mathrm{c}^{2}\right)$ is totally electromagnetic, which was the original idea of Lorentz and Abraham. This is consistent with the (nearly, if not exactly) zero rest mass of the electron's uncharged weak isodoublet partner-the neutrino-and with order of magnitude of the neutron-proton mass difference $\left(1.29 \mathrm{MeV} / \mathrm{c}^{2}\right)$. There is no need to invoke any nonelectromagnetic forces within the electroncollectively known as Poincaré stresses.

The energy of an electromagnetic field in a rest frame is given by

$$
W=\frac{1}{8 \pi} \int(\mathbf{E} \cdot \mathbf{D}+\mathbf{B} \cdot \mathbf{H}) d^{3} \mathbf{r} .
$$

The field produced by a point charge $e$ in vacuum is represented by $\mathbf{D}=\mathbf{E}=e \hat{\mathbf{r}} / r^{2}, \mathbf{B}=\mathbf{H}=0$ and

$$
W=\frac{1}{8 \pi} \int \frac{e^{2}}{r^{4}} 4 \pi r^{2} d r=\infty,
$$

unless a lower cutoff is introduced.

It was suggested a long time ago by Furry and Oppenheimer [11] that quantum electrodynamic (QED) effects could give the vacuum some characteristics of a polarizable medium, which Weisskopf [12] represented phenomenologically by an inhomogeneous dielectric constant, viz

$$
\mathbf{D}(r)=\epsilon(r) \mathbf{E}(r) \text {. }
$$

Constitutive relations in classical electrodynamics describe properties of matter which must be determined experimentally or, in favorable cases, by quantum-theoretical computation. In the same sense, Eq. (4) represents a constitutive relation for the vacuum, as implied by quantum electrodynamics. Using (4) in (2),

$$
W=\frac{1}{8 \pi} \int_{0}^{\infty} \frac{1}{\epsilon(r)} \frac{e^{2}}{r^{4}} 4 \pi r^{2} d r
$$

and equating this to the self-energy of the electron

$$
W=\frac{e^{2}}{2} \int_{0}^{\infty} \frac{d r}{r^{2} \epsilon(r)}=m c^{2} .
$$

Remarkably, the functional form of $\epsilon(r)$ need not be further specified, provided only that it satisfies the limiting conditions

$$
\epsilon(\infty)=1 \quad \text { and } \quad \epsilon(0)=\infty .
$$

Maxwell's first equation $\nabla \cdot E=4 \pi \rho$ applied to the electric field

$$
\mathbf{E}=\frac{e \mathbf{r}}{\epsilon(r) r^{3}}
$$

determines the charge density

$$
\rho(r)=-\frac{e \epsilon^{\prime}(r)}{4 \pi r^{2}[\epsilon(r)]^{2}} .
$$

Note that this represents the net or total charge density, the sum of the free and polarization densities. 


\section{BLINDER}

This function is appropriately normalized since

$$
\begin{aligned}
\int_{0}^{\infty} \rho(r) 4 \pi r^{2} d r & =-e \int_{0}^{\infty} \frac{\epsilon^{\prime}(r) d r}{[\epsilon(r)]^{2}} \\
& =e\left[\frac{1}{\epsilon(\infty)}-\frac{1}{\epsilon(0)}\right]=e .
\end{aligned}
$$

An explicit functional form for $\epsilon(r)$ does follow if it is conjectured that the net charge density (9) is proportional to the field energy density from (6). For then,

$$
\frac{\epsilon^{\prime}(r)}{\epsilon(r)}=-\frac{e^{2}}{2 m c^{2} r^{2}}
$$

with the solution

$$
\epsilon(r)=\exp \left(\frac{e^{2}}{2 m c^{2} r}\right)=\exp \left(\frac{r_{0}}{2 r}\right) .
$$

It should be emphasized for the benefit of QED theorists who might be reading this that our use of the term "vacuum polarization" is intended only in a classical phenomenological context. The leading contribution to vacuum polarization in real life comes from the interaction of the electron with the transverse radiation field, which does not enter in our model. We are thereby overlooking additional self-energy contributions arising from fluctuations hi the vacuum radiation field. Accordingly, our representation of vacuum polarization is not to be compared with QED computations.

Somewhat of a rationalization for the functional form of $\epsilon(r)$ is suggested by Debye-Hückel theory for ionic solutions and plasmas. The dielectric constant depends on a Boltzmann factor $e^{-\mathcal{E} / k T}$. If in place of the average thermal energy $k T$, we substitute the relativistic energy of pair formation $2 m c^{2}$, regarding the vacuum as an effective thermal reservior, then Eq. (12) follows with $\mathcal{E}=e^{2} / r$.

An explicit expression for the charge density follows by substituting (12) into (9):

$$
\rho(r)=\frac{e r_{0}}{8 \pi r^{4}} e^{-r_{0} / 2 r} .
$$

Since $\rho_{\text {free }}(r)=e \delta(\mathbf{r})$, the density from vacuum polarization must equal

$$
\rho_{\mathrm{VP}}(r)=\frac{e r_{0}}{8 \pi r^{4}} e^{-r_{0} / 2 r}-e \delta(\mathbf{r}),
$$

which is represented graphically in Figure 1. According to this model, the free point charge is exactly canceled by the delta function term of the polarization charge. This is certainly reminiscent of the self-energy cancellation that occurs in Eq. (1).

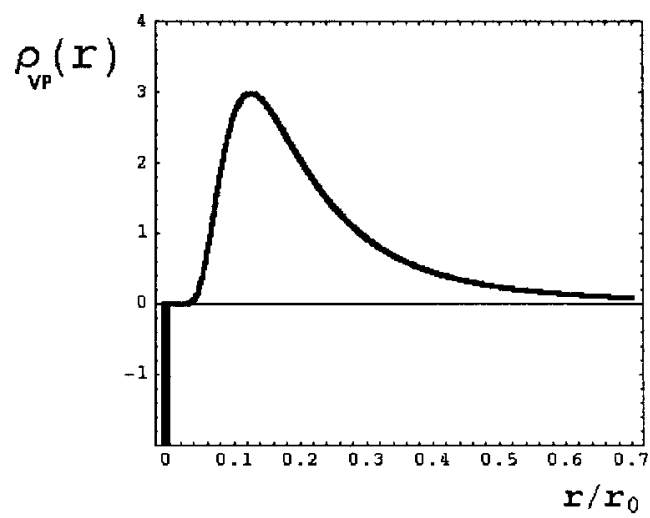

FIGURE 1. Vacuum polarization produced by a point charge. The vertical bar at $r=0$ represents a delta function, which cancels the free charge.

The electrostatic potential corresponding to (14) is given by

$$
\Phi(\mathbf{r})=\frac{2 e}{r_{0}}\left(1-e^{-r_{0} / 2 r}\right) .
$$

This implies a deviation from Coulomb's law of the same magnitude as the fine structure in atoms, but totally negligible on a macroscopic scale reality such as the Lamb shift. Note that (15) reduces to $e / r$ when either $r_{0} \rightarrow 0$ or $r \rightarrow \infty$.

An alternative evaluation of the electromagnetic self-energy follows from transformation of Eq. (2):

$$
W=\frac{1}{8 \pi} \int \mathbf{E} \cdot \mathbf{D} d^{3} \mathbf{r}=\frac{1}{2} \int \Phi_{\text {free }} \rho d^{3} \mathbf{r}
$$

using

$$
\mathbf{D}=-\nabla \Phi_{\text {free }}=\frac{e \mathbf{r}}{r^{3}}
$$

and assuming the requisite vanishing of integrands at infinity. Thus

$$
\begin{aligned}
W & =\frac{1}{2} \int_{0}^{\infty} \Phi_{\text {free }}(r) \rho(r) 4 \pi r^{2} d r \\
& =\frac{e^{2} r_{0}}{4} \int_{0}^{\infty} \frac{e^{-r_{0} / 2 r}}{r^{3}} d r=m c^{2}
\end{aligned}
$$

in agreement with the previous result, and further justification for the conjectured functional form of $\epsilon(r)$.

We have also been able to obtain the result of this study by an alternative derivation from the viewpoint of general relativity [13]. A modification of the Reissner-Nordstrøm solution to the EinsteinMaxwell equations has been shown to give a finite electron self-energy. 


\section{References}

1. See Preface to any of the early issues of Advances in Quantum Chemistry; Academic Press: New York; for example, Vol. 2, 1965.

2. Thomson, J. J. Phil Mag 1897, 44, 293.

3. For a retrospective on the electron centennial, see Weinberg, S. Nature 1997, 386, 213.

4. A definitive review of classical electron theories is given by Rohrlich, F. Classical Charged Particles; Addison-Wesley: Reading, MA, 1990.

5. See, for example, Perkins, D. H. Introduction to High Energy Physics; Addison-Wesley: Reading, MA, 1987.
6. Dirac, P. A. M. Proc Roy Soc (London) 1938, A167, 148.

7. Wheeler, J. A.; Feynman, R. P. Rev Mod Phys 1949, 17, 157; $1945,21,425$.

8. Rohrlich, F. Phys Rev Lett 1964, 12, 375.

9. Teitelboim, C. Phys Rev D 1970, 1, 1572; 1970, 3, 297; 1971, 4,345 .

10. Frenkel, J. Zeit Phys 1925, 32, 518.

11. Furry, W.; Oppenheimer, J. R. Phys Rev 1934, 45, 245.

12. Weisskopf, V. F. Det Kgl Danske Videnskab Selskab MatFys Medd 1936, 14, 1; Reprinted in Schwinger, J. Quantum Electrodynamics; Dover: New York, 1958.

13. Blinder, S. M. Repts Math Phys 2001, 47, 279. 